\section{Polimorfismos de genes de glutatión S-transferasa M1 y T1 como factores pronósticos del cáncer de vejiga: evidencia limitada e inconsistencias que aún no se resuelven}

\section{Glutathione S-transferase T1 and M1 gene polymorphisms as prognostic factors for bladder cancer: limited evidence and still unresolved inconsistencies}

Meng $X Y^{1}{ }^{1}$ Shi $\mathrm{MJ}^{2}$
El cáncer de vejiga es un tumor maligno común y los factores genéticos pueden desempeñar un papel importante en su manifestación, recurrencia y progresión. La relación entre el riesgo de cáncer de vejiga y los polimorfismos genéticos de glutatión S-transferasa (GST) T1 y M1 se han discutido en metanálisis recientes; ${ }^{1}$ sin embargo, actualmente no existe un resumen completo de su importancia pronóstica. En los estudios primarios se observan hallazgos inconsistentes; por tanto, realizamos una revisión bibliográfica de este tema, para resumir la información disponible.

Se realizó una búsqueda exhaustiva en la base de datos PubMed. Después de la selección por relevancia, se incluyeron nueve estudios publicados entre 2005 y 2015, en los que evaluaron pacientes con cáncer de vejiga y la relación entre los polimorfismos de GSTT1/GSTM1 (nulo vs positivo) y el pronóstico. ${ }^{2-10}$ Tres estudios incluyeron pacientes con cáncer de vejiga super-

\author{
${ }^{1}$ Centro para la Medicina Basada en Evidencia y la \\ Medicina Translacional, Hospital Zhongnan de la \\ Universidad de Wuhan, China. \\ 2 Institut Curie, PSL Research University, CNRS, UMR \\ 144, París, Francia. \\ Recibido: junio 2017 \\ Aceptado: junio 2017 \\ Correspondencia \\ Meng Xiang Yu \\ mengxy_whu@163.com
}

Este artículo debe citarse como

Meng XY, Shi MJ. Polimorfismos de genes de glutatión S-transferasa M1 y $\mathrm{T} 1$ como factores pronósticos del cáncer de vejiga: evidencia limitada e inconsistencias que aún no se resuelven. Rev Mex Urol. 2017 jul-agos; 77(4):336-338.

DOI: https://doi.org/10.24245/revmexurol.v77i4.1477 
ficial (CVS) e invasivo (CVI), ${ }^{2,3,6}$ cuatro estudios se centraron solamente en $\mathrm{CVS}^{4,5,9,10}$ y dos en CVI. ${ }^{7,8}$ Se encontraron cinco investigaciones coreanas $^{4,5,8,9}$ y cuatro ensayos efectuados con pacientes caucásicos. ${ }^{3,6,7,10}$ El primer estudio, realizado en 153 pacientes con cáncer de vejiga, no mostró repercusión pronóstica significativa del polimorfismo de GSTT1, pero el genotipo GSTM1-positivo aumentó más de tres veces el riesgo de evolución de la enfermedad $(p=0.016){ }^{2}$ La primera investigación relevante en sujetos de raza caucásica fue publicada en 2010, que reportó 1.9 veces más riesgo de mortalidad en pacientes con GSTM1-negativo $(p=0.05) .{ }^{3} \mathrm{Ha}$ y sus coautores examinaron sólo pacientes con CVS e informaron que el genotipo GSTT1-positivo se asocia con mayor riesgo de recurrencia $(p=0.043)$ y progresión $(p=0.006)$, de 1.6 y 3.4 veces, respectivamente; y el genotipo GSTM1negativo se relaciona con 2.7 mayor riesgo de recurrencia $(p=0.031) .4$ En 2012, Roth y sus colegas publicaron un estudio con 213 pacientes alemanes con cáncer de vejiga y encontraron que la positividad de GSTT1 disminuyó 40\% el riesgo de recurrencia $(p=0.007)$ y el GSTM1 no influyó significativamente en esta variable $(p=0.36) .{ }^{6}$ El estudio de Djukic y su grupo (2013), en el que informaron hallazgos de pacientes serbios con cáncer de vejiga invasivo, reportó que el genotipo GSTT1-positivo representó un factor de riesgo de mala supervivencia global $(p=0.028)$, pero la repercusión del polimorfismo de GSTM1 no fue significativo $(p=0.694) .{ }^{7}$ Por su parte, Kang y sus coautores analizaron la importancia pronóstica de los polimorfismos de genes de GSTT1 y GSTM1 en pacientes con cáncer de vejiga superficial o invasivo, y encontraron que en los sujetos con cáncer de vejiga invasivo, el genotipo GSTT1-nulo aumentó 3 veces más el riesgo de progresión y supervivencia cáncer-específica ( $p=0.001$ y 0.003 , respectivamente), sin efecto significativo en el polimorfismo de GSTM1. En los pacientes con cáncer de vejiga superficial, la positividad de GSTT1 se correlacionó con mayor riesgo de recurrencia $(p=0.038) .^{8,9} \mathrm{El}$ estudio más reciente, es decir, el de Lacombe y colaboradores, no reportó diferencias significativas de recurrencia de cáncer entre los genotipos GSTM1-nulo y GSTM1-positivo en un grupo de pacientes canadienses con cáncer de vejiga superficial. ${ }^{10}$ La Figura 1 muestra la información al respecto.

Hasta la fecha existen pocos datos relacionados con este tema y encontramos gran inconsistencia entre los resultados de los diferentes estudios. No se determina la importancia pronóstica exacta de los polimorfismos de GSTM1 y GSTT1 para el cáncer de vejiga. La diferencia entre el tipo de neoplasia (cáncer superficial o invasivo) y el origen étnico (asiático, caucásico) puede explicar esta heterogeneidad. Por el momento no puede establecerse ninguna conclusión al respecto, pues los datos disponibles son escasos. Se requieren investigaciones y evaluaciones adicionales, con un tamaño de muestra adecuado, diseño riguroso, análisis exhaustivo y un informe estándar de los resultados.

\section{REFERENCIAS}

1. Yu C, Hequn C, Longfei L, et al. GSTM1 and GSTT1 polymorphisms are associated with increased bladder cancer risk: Evidence from updated meta-analysis. Oncotarget. 2017;8(2):3246-58.

2. Kim EJ, Jeong P, Quan C, et al. Genotypes of TNF-alpha, VEGF, hOGG1, GSTM1, and GSTT1: useful determinants for clinical outcome of bladder cancer. Urology. 2005;65(1):70-5.

3. Norskov MS, Frikke-Schmidt R, Bojesen SE, et al. Copy number variation in glutathione-S-transferase $\mathrm{T} 1$ and M1 predicts incidence and 5-year survival from prostate and bladder cancer, and incidence of corpus uteri cancer in the general population. Pharmacogenomics J. 2011;11(4):292-9.

4. Ha YS, Yan C, Lym MS, et al. GSTT1 as a prognosticator for recurrence and progression in patients with non-muscleinvasive bladder cancer. Dis Markers. 2010;29(2):81-7.

5. Ha YS, Yan C, Jeong P, et al. GSTM1 tissue genotype as a recurrence predictor in non-muscle invasive bladder cancer. J Korean Med Sci. 2011;26(2):231-6.

6. Roth E, Selinski S, Schikowsky C, et al. Bladder cancer survival in a former industrial area in Saxony-Anhalt, Germany. J Toxicol Environ Health A. 2012;75(19-20):1216-25. 
7. Djukic TI, Savic-Radojevic AR, Pekmezovic TD, et al. Glutathione S-transferase $\mathrm{T} 1, \mathrm{O} 1$ and $\mathrm{O} 2$ polymorphisms are associated with survival in muscle invasive bladder cancer patients. PloS One. 2013;8(9):e74724.

8. Kang HW, Song PH, Ha YS, et al. Glutathione S-transferase M1 and T1 polymorphisms: susceptibility and outcomes in muscle invasive bladder cancer patients. Eur J Cancer. 2013;49(14):3010-9.
9. Kang HW, Tchey DU, Yan C, et al. The predictive value of GSTT1 polymorphisms in predicting the early response to induction BCG therapy in patients with non-muscle invasive bladder cancer. Urol Oncol. 2014;32(4):458-65.

10. Lacombe L, Fradet V, Levesque $\mathrm{E}$, et al. Phase II Drug-Metabolizing Polymorphisms and Smoking Predict Recurrence of Non-Muscle-Invasive Bladder Cancer: A Gene-Smoking Interaction. Cancer Prev Res (Phila). 2016;9(2):189-95.

A

Auto

Kim y col. Ambos

Nørskov y col. Ambos

Ha y col. NMIBC

Ha y col. NMIBC

Roth y col. Ambos

Djukic y col. MIBC

Kang y col. MIBC

Kang y col. NMIBC

Lacombe y col. NMIBC

B

\section{Autor}

Kim y col.

Nørskov y col.

Ha y col.

Ha y col.

Roth y col.

Djukic y col.

Kang y col

Kang y col.

Lacombe y col.
Tipo

Ambos

Ambos

NMIBC

NMIBC

Ambos

MIBC

MIBC

NMIBC

NMIBC
Raza

Asiática

Caucásica

Asiática

Asiática

Caucásica

Caucásica

Asiática

Asiática

Caucásica

\section{Recurrencia}

Evolución

Supervivencia

\begin{tabular}{|l|l|l|}
\hline & & \\
\hline & & \\
\hline & & \\
\hline & & \\
\hline & & \\
\hline & & \\
\hline & & \\
\hline & & \\
\hline & & \\
\hline
\end{tabular}

GSTT1

Raza

Recurrencia

Evolución

Supervivencia

Asiática

Caucásica

Asiática

Asiática

Caucásica

Caucásica

Asiática

Asiática

Caucásica

\begin{tabular}{|l|l|l|}
\hline & & \\
\hline & & \\
\hline & & \\
\hline & & \\
\hline & & \\
\hline & & \\
\hline & & \\
\hline & & \\
\hline & & \\
\hline
\end{tabular}

Figura 1. Visualización de la información incluida. A: asociación entre el polimorfismo de GSTM1 y el pronóstico; B: asociación entre el polimorfismo de GSTT1 y el pronóstico. Color rojo: favorece el genotipo-nulo; verde: favorece el genotipo-positivo; gris: sin asociación significativa; blanco: no informado.

NMIBC: cáncer de vejiga no invasor a músculo (superficial); MIBC: cáncer de vejiga invasor a músculo (invasivo); GSTM1: polimorfismo de glutatión S-transferasa M1; GSTT1: polimorfismo de glutatión S-transferasa T1. 